\title{
Anabases
}

ANABASES Traditions et réceptions de l'Antiquité

$21 \mid 2015$

Varia

\section{La paideia à la croisée des humanismes : Marrou lecteur de Jaeger}

\section{Anthony Andurand}

\section{(2) OpenEdition}

12 Journals

Édition électronique

URL : http://journals.openedition.org/anabases/5331

DOI : 10.4000/anabases.5331

ISSN : 2256-9421

Éditeur

E.R.A.S.M.E.

Édition imprimée

Date de publication : 1 avril 2015

Pagination : 231-236

ISSN : 1774-4296

\section{Référence électronique}

Anthony Andurand, "La paideia à la croisée des humanismes : Marrou lecteur de Jaeger », Anabases [En ligne], 21 | 2015, mis en ligne le 01 avril 2018, consulté le 21 octobre 2019. URL : http:// journals.openedition.org/anabases/5331 ; DOI : 10.4000/anabases.5331 


\section{La paideia à la croisée des humanismes: Marrou lecteur de Jaeger}

Anthony ANDurand

U n «événement considérable ${ }^{1} »$ : c'est ainsi que Marrou, dans un long compte rendu publié en I946 dans les mélanges de la Revue historique, salue la parution des deuxième et troisième volumes de Paideia, produit des recherches menées par l'helléniste Werner Jaeger², depuis une vingtaine d'années, sur l'histoire de la littérature et de la culture grecques. Il est vrai que le monde de l'érudition, alors, attend depuis quelque temps déjà l'achèvement de cette imposante étude, due à l'un des maîtres de la science allemande et dont le premier tome ${ }^{3}$, publié en ig34, a été fort bien accueilli ${ }^{4}$.

Presque dix ans ont passé lorsque le philologue fait paraître, respectivement en I943 et I944, les deux derniers volets de son opus magnum ${ }^{5}$. C'est aux presses

1 H.-I. Marrou, “Le siècle de Platon. À propos d'un livre récent », Revue Historique ig6 (I946), p. I/22-I49, p. I/42.

2 Pour une présentation synthétique du parcours et de l'œuvre de Werner Jaeger (I888-I96I): W.M. Calder, “Werner Jaeger », in W.M. Calder, W.Briggs (ed.), Classical Scholarship. A biographical Encyclopedia, New York, Londres, Garland Publishing, I990, p. 2II-226.

3 W. Jaeger, Paideia. Die Formung des griechischen Menschen, Berlin, W. de Gruyter, I934.

4 Sur la composition du premier volume de Paideia et son accueil dans le monde des études classiques: B.NäF, "Werner Jaegers Paideia: Entstehung, kulturpolitische Absichten und Rezeption ", in W. M. CALDER (ed.), Werner Jaeger Reconsidered, Atlanta, Scholars Press, I992, p. I25-146.

5 W. JAEger, Paideia. The Ideals of Greek Culture. II: In Search of the Divine Centre, New York, Oxford University Press, I943; III: The Conflict of CulturalIdeals in the Age of Plato, New York, Oxford University Press, I944. Les deux volumes sont par la suite publiés en 
universitaires d'Oxford et en anglais, du reste, d'après une traduction du manuscrit allemand préparée par Gilbert Highet ${ }^{6}$, que les deux volumes ont été publiés, sous le titre Paideia: The Ideals of Greek Culture. Indirectement touché par les lois raciales ${ }^{7}$, Jaeger avait en effet dû quitter Berlin en Ig36 pour rallier les États-Unis et l'université de Chicago, avant de rejoindre, en I939, la prestigieuse université de Harvard, où il enseigna jusqu'à sa mort en I96r.

Si le texte de Marrou, connu de quelques commentateurs ${ }^{8}$, ne saurait être considéré comme un “classique» de l'érudition, il mérite qu'on lui fasse une place dans les annales des sciences de l'Antiquité. La recension est, d'abord, un modèle du genre: on y retrouve, en quelques pages serrées, cette hauteur de vue soucieuse de dégager, de l'examen des faits concrets du passé, des problèmes encore pertinents pour la culture du temps présent; ce style patient, aussi, et ce ton audacieux, qui assume volontiers l'analogie historique ${ }^{9}$ comme un outil d'intelligibilité à l'usage de l'historien et de ses contemporains. De fait, le compte rendu de Marrou est une lecture lumineuse de Paideia, en même temps qu'une excellente introduction à l'œuvre de Jaeger. Le portrait dressé au fil des pages révèle sa parfaite connaissance des travaux du philologue allemand, depuis la thèse sur Aristote (Igı2) jusqu'à ses recherches récentes sur la culture grecque et les origines du christianisme, en passant par son volume dédié à Démosthène (I938); Marrou n’ignore pas, non plus, les efforts entrepris par Jaeger, au sein de l'école qu'il avait su regrouper autour de sa personne et de la revue Die Antike, pour inspirer dans l'Allemagne weimarienne un nouvel humanisme, le «troisième» (dritter Humanismus), capable de placer les valeurs idéales de l'héritage grec et du génie platonicien au service de la communauté politique et de l'action humaine. “On sait quelle noble ambition, suggère Marrou dans sa recension, anime l'œuvre de ce grand éducateur qu'est W.Jaeger:

allemand, chez W. de Gruyter, respectivement en I944 et I947. L'édition recensée par Marrou est la deuxième édition anglaise, parue chez Blackwell en I945.

6 Classiciste et professeur à Columbia, Gilbert Highet (ı9o6-ı978) est par ailleurs l'auteur de l'ouvrage The Classical Tradition: Greek andRoman Influences on Western Literature, Oxford, New York, Oxford University Press, I949.

7 Jaeger était alors marié, depuis I93I, à Ruth Heinitz, qui fut, à la fin de l'année I935, déchue de la nationalité allemande par les Lois de Nuremberg.

8 On signalera ici la contribution de P.Demont, «H.-I. Marrou et les “deux colonnes du temple" : Isocrate et Platon », in J.-M.PaIller, P. Payen (dir.), Que reste-t-il de l'éducation classique? Relire "le Marrou», Histoire de l'éducation dans l'Antiquité, Toulouse, Presses universitaires du Mirail, 2004, p. Iog-IIg (le texte de Marrou est analysé p. IIo-III).

9 Ainsi Jaeger, ayant quitté l’Allemagne et trouvé refuge aux États-Unis «comme les savants byzantins du $\mathrm{Xv}^{\mathrm{e}}$ siècle l'avaient cherché en Italie» (p. I/2) ; l'évocation du jeune Aristote, "privat-docent de rhétorique à l'Académie», et le portrait d'Isocrate, "porteparole de la nouvelle droite, disons de la droite constitutionnelle modérée» (p. I/4). 
humaniste, il ne sépare pas la connaissance de la vie ${ }^{10}$. Empreint d'estime, l'hommage ne cède pourtant rien à la lucidité, et n'élude pas les affinités qui ont un temps rapproché l'historien, “honneur de la science allemande», "victime de la barbarie nouvelle», de «l'esprit nouveau que les nazis faisaient triompher ${ }^{\prime \prime}$ ».

C'est donc à la lumière de l'engagement humaniste de Jaeger, écho de ses propres préoccupations, que Marrou se propose de relire Paideia. La recension se présente comme un document historiographique de première importance, témoignage sur le vif de la lente maturation, au contact des questionnements et des renouvellements de la science contemporaine, d'une pensée ample et originale. Dans la continuité d'une réflexion amorcée depuis plusieurs années, Marrou s'y montre particulièrement attentif à la démarche de son homologue allemand et aux questions de méthode qu'elle tend à soulever. L'étude de Jaeger est ainsi décrite comme l'exemple le plus remarquable du «nouvel esprit historique ${ }^{12}$ », cette attitude qui, prenant appui sur les données événementielles et concrètes de l'histoire, s'applique à dégager des témoignages du passé des «idées » et des “valeurs » valables pour “l'homme vivant ${ }^{13}$ ». De fait, souligne Marrou, Jaeger ne s'intéresse guère, dans son étude sur la paideia, aux pratiques, aux méthodes et aux contenus de l'éducation dans le monde grec - aspects auxquels l'auteur de l'Histoire de l'éducation dans l'Antiquité se montrera plus sensible. Paideia peut davantage être envisagé comme une histoire de la littérature grecque, d'Homère à Démosthène, abordée sous l'angle de sa portée éducative et formatrice. Dans ce vaste tableau, insiste par ailleurs Marrou, “l'essentiel de l'effort est consacré à Platon ${ }^{14}$ ». Le premier volume de Paideia, qui traitait de la période archaïque et du v siècle athénien, s'achevait par un chapitre consacré à Thucydide, le «penseur politique» (politischer Denker); les deuxième et troisième volumes - soit près de 750 pages -, en revanche, sont pour les deux tiers dédiés à la philosophie platonicienne. Le lecteur, remarque ainsi fort justement le recenseur, peut légitimement se demander «si le véritable sujet du livre n'est pas une initiation à l'étude de Platon ${ }^{15}$ ».

10 H.-I. Marrou, “Le siècle de Platon », p. Í42.

11 Ibid., p.I42. Sur les rapports équivoques de Jaeger avec l'idéologie nationale-socialiste et ses tentatives pour se rapprocher du nouveau régime entre ig33 et I936, on pourra consulter la mise au point de W. M. CALdER, “Werner Jaeger and Richard Harder: an Erklärung», $Q S$ I7 (I983), p. 99-I2I.

12 La formule apparaît dans le titre d'un article donné au Monde le i2 juillet de la même année.

13 H.-I. Marrou, «Le siècle de Platon », p. I433.

14 Ibid., p. 145 .

15 Ibid., p. I44. Notons toutefois qu'il s'agit là, de la part de l'auteur de Paideia, d'un choix délibéré et assumé: dans la préface de l'édition anglaise, Jaeger n'hésite pas à présenter son étude comme une «introduction à Platon », décrit comme «le véritable philosophe 
L'ouvrage, dès lors, prend place dans la grande tradition des études platoniciennes - "forme moderne de la permanence du platonisme même ${ }^{16}$ »-, dont la science allemande, de Schleiermacher à Stenzel ${ }^{17}$, s'est fait une spécialité. L'ombre du Platon de Wilamowitz accompagne le lecteur: avec Paideia, observe Marrou, Jaeger livre à l'érudition une "grande synthèse» sur le philosophe, «analogue et rivale $^{18}$ » à celle du maître. Analogue, parce que Jaeger se propose de retracer, comme Wilamowitz et comme il l'a déjà fait pour Aristote, l'itinéraire spirituel de Platon, tel qu'il se révèle dans la succession des dialogues et dans le témoignage autobiographique de la Lettre VII, tenu pour authentique; rivale, cependant, car, contrairement à son prédécesseur, soucieux de dégager les étapes du développement intérieur (innere Entwicklung) et de l'élaboration de la pensée platonicienne, l'auteur de Paideia, lui, souligne à l'inverse “l'unité profonde de tout Platon», animé par la conviction que l'éducation ne constitue pas un aspect, parmi d'autres, de l'œuvre du philosophe, mais bien plutôt «l'essence même du génie platonicien $^{19}$ ». Sur ce dernier point, enfin, alors que le Platon de Wilamowitz, en trouvant refuge dans les murs paisibles de l'Académie, n'était devenu pédagogue que sous l'effet des circonstances extérieures et d'expériences déçues, celui de Jaeger, au contraire, des premiers dialogues à la République et aux Lois, se fait éducateur comme par vocation, guidé par les visées pratiques d'une philosophie au service de la cité et de l'ambition réformatrice ${ }^{20}$.

de la paideia (W.JAEger, "Preface », Paideia. The Ideals of Greek Culture. II: In Search of the Divine Centre, Oxford, Blackwell, I947, p. x-XI).

Ibid., p. 146 .

17 J. Stenzel, Platon der Erzieher, Leipzig, F. Meiner, I928 (et non ı938, comme indiqué, de manière erronée, dans la recension de Marrou).

18 H.-I. Marrou, “Le siècle de Platon », p. I45.

19 Ibid., p. I47.

20 Sur ce point, du reste, il n'est pas certain, comme le suggère Marrou dans son compte rendu (p.148), que le Platon de Jaeger, "parti pour construire une cité » aboutisse «à rêver d'une Église». Cette lecture "personnaliste» de Paideia, par ailleurs reprise deux ans plus tard dans une note de l'Histoire de l'éducation dans l'Antiquité (Paris, Seuil, 2 vol., I9917 [1948], I, p.367, n. 20), paraît quelque peu excessive. Chez Jaeger, la philosophie de Platon, dans les dernières années de la vie de celui-ci, oscille en effet entre l'ambition pratique d'une refonte de la cité et la tentation d'une sagesse toute personnelle, contemplative et dégagée des sollicitations politiques - le repli du philosophe "vers la cité intérieure qu'il porte en lui-même» (Platon, Rép., IX, 5gie:

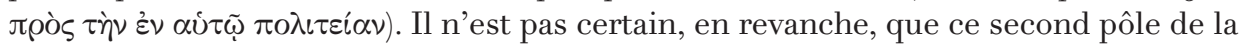
philosophie platonicienne, dans la présentation de Jaeger, prenne définitivement le pas sur le projet politique initial: pour le philologue allemand, celle-ci demeure jusqu'au bout - autrement dit, jusqu'à la composition des Lois - une philosophie de l'action, animée par l'effort de rénovation de la vie politique et de la cité grecques. 
Délaissant le champ des études platoniciennes et les traditions de la science allemande, la dernière partie du compte rendu s'efforce d'évaluer l'apport de Paideia à la compréhension de l'histoire culturelle du monde grec. C'est à ce stade que l'analyse de Marrou manifeste ses divergences avec l'approche et les conceptions jaegeriennes, laissant ainsi entrevoir ce que seront les orientations de la future Histoire de l'éducation dans l'Antiquité, alors en préparation ${ }^{21}$. La ligne de partage s'insinue d'abord, pourrait-on dire, dans le choix de la période de référence. Jaeger a choisi de placer au centre de son enquête le $\mathrm{Iv}^{\mathrm{e}}$ siècle athénien “cette période attachante, ajoute le recenseur, à mesure qu'elle nous est mieux connue $^{22}$ », et fait de “l'âge de Platon ${ }^{23}$ »'époque “classique de l'histoire de la paideia $^{24}$ ». C'est “sous un autre éclairage ${ }^{25}$ », cependant, que Marrou souhaite appréhender le devenir de l'éducation antique: «Je situerais volontiers à l’époque hellénistique (prolongée jusqu'à la fin du Haut-Empire romain: je crois à l'unité de l'hellenistisch-römische Kultur) l'akmè de la paideia antique, l'apogée de l'art de modeler la forme intérieure de la personnalité humaine ${ }^{26}$. „En proposant d'élargir la perspective chronologique, Marrou n'invite pas seulement le spécialiste à envisager dans la longue durée l'évolution de l'éducation et de la culture grecques; le programme de revalorisation de la période hellénistique - c'est ici que son analyse marque un nouvel écart par rapport à l'étude de Jaeger - doit aussi être compris comme une redéfinition du contenu même de la paideia et des références auxquelles elle puise: “Par contre-coup, suggère-t-il, j’en viendrais à revaloriser, vis-à-vis de Platon, le rôle historique d'Isocrate, le grand initiateur au point de vue des institutions pédagogiques, le fondateur de la grande tradition de l'école antique $^{27}$.» Confiant dans la portée et la valeur du «message » platonicien pour le temps présent, Jaeger a peut-être accordé une place excessive aux leçons du philosophe dans son histoire de la paideia. Car, dans la lecture proposée par Marrou, Platon fait bien figure, au risque d'une certaine audace, de “vaincu » de l'histoire:

21 Dans De la connaissance historique, Marrou confie en effet que le livre lui a été “commandé» en I943 par un “éditeur ami» des Éditions du Seuil (De la connaissance historique, Paris, Le Seuil, I9757 [1954], p. 202).

22 H.-I. Marrou, «Le siècle de Platon », p. I44.

23 L'expression apparaît dans le sous-titre du troisième volume de Paideia.

24 W. JAEger, “Preface », Paideia, II, p. xI.

25 H.-I. Marrou, «Le siècle de Platon », p. I48.

26 Ibid., p. I49.

27 Ibid., p.I49. Ces positions, remarque à juste titre Paul Demont, se voient quelque peu infléchies dans l'Histoire de l'éducation dans l'Antiquité, où Platon et Isocrate, dans les chapitres qui leur sont consacrés, sont désormais décrits, selon la formule bien connue, comme «les deux colonnes du temple», "se faisant équilibre et comme se répondant» (P.Demont, «H.-I. Marrou et les “deux colonnes du temple” », p. III). 
ce n'est pas l'enseignement platonicien, mais celui d'Isocrate et de la tradition rhétorique que les générations ultérieures ont suivi et reçu pour donner vie à la “tradition antique», cette tradition, prend soin de préciser l'historien pour finir, dont toute une civilisation a vécu et « dont nous vivons encore ${ }^{28}$ ».

Le compte rendu de Marrou conduit ainsi le lecteur aux origines mêmes de cet humanisme dont l'historien n'a cessé de se revendiquer et de sonder les sources historiques et spirituelles. Sans doute la recherche de ces dernières décennies a-t-elle fait éclater les cadres de l'humanisme traditionnel et ébranlé ses certitudes, cependant que les pérégrinations de la notion, depuis les errements du programme jaegerien jusqu'aux usages plus récents du terme, contribuaient à en affaiblir la portée. Plus d'un demi-siècle après la parution de Paideia et de l'Histoire de l'éducation, toutefois, le texte de Marrou conserve remarquablement la trace de cet effort pour interroger et embrasser, au présent, dans la patiente fréquentation des Anciens et le dialogue engagé avec les contemporains, les voies et les détours d'une tradition.

\author{
Anthony Andurand \\ PLH-ERASME (EA 4601) \\ Université Toulouse-Jean Jaurès \\ Pavillon de la Recherche \\ 5, allées Antonio Machado \\ F-31058 Toulouse Cedex 9 \\ anthonyandurand@yahoo.fr
}

28 H.-I. Marrou, “Le siècle de Platon », p. I49. 